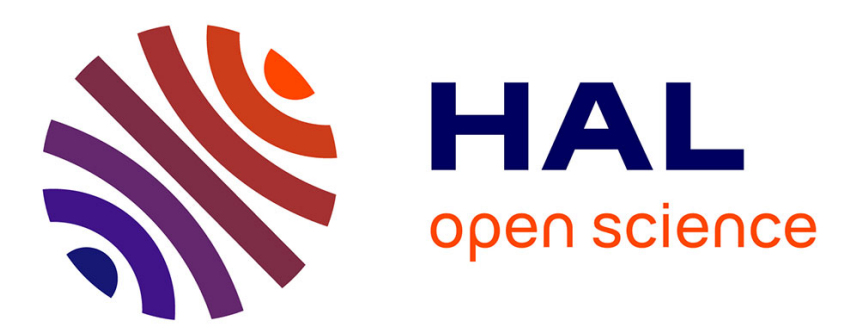

\title{
System performances of fiber optical parametric amplifiers
}

T. Sylvestre, Arnaud Mussot, A. Vedadi, L. Provino, E. Lantz, H. Maillotte

\section{To cite this version:}

T. Sylvestre, Arnaud Mussot, A. Vedadi, L. Provino, E. Lantz, et al.. System performances of fiber optical parametric amplifiers. Fiber and Integrated Optics, 2009, 27 (6), pp.516-531. 10.1080/01468030802269787 . hal-00460181

\section{HAL Id: hal-00460181 \\ https://hal.science/hal-00460181}

Submitted on 28 Aug 2013

HAL is a multi-disciplinary open access archive for the deposit and dissemination of scientific research documents, whether they are published or not. The documents may come from teaching and research institutions in France or abroad, or from public or private research centers.
L'archive ouverte pluridisciplinaire HAL, est destinée au dépôt et à la diffusion de documents scientifiques de niveau recherche, publiés ou non, émanant des établissements d'enseignement et de recherche français ou étrangers, des laboratoires publics ou privés. 


\title{
System Performances of Fiber Optical Parametric Amplifiers
}

\author{
THIBAUT SYLVESTRE, ${ }^{1}$ ARNAUD MUSSOT,,${ }^{1, *}$ \\ ARMAND VEDADI, ${ }^{1, \dagger}$ LAURENT PROVINO,, , \\ ERIC LANTZ, ${ }^{1}$ and HERVÉ MAILLOTTE ${ }^{1}$ \\ ${ }^{1}$ Département d'Optique P. M. Duffieux, Institut FEMTO-ST, Centre National \\ de la Recherche Scientifique UMR 6174, Université de Franche-Comté, \\ Besançon, France
}

\begin{abstract}
The research field of fiber optical parametric amplifiers has steadily expanded over the last two decades as a host of all-optical signal processing techniques have been demonstrated in nonlinear optical fibers such as wavelength conversion, optical regeneration, optical switching, limiting, buffering, and sampling. This article reviews the system performances of theses parametric devices such as gain bandwidth, focuses on the main limitations and demonstrates efficient techniques for suppressing them.
\end{abstract}

Keywords amplifiers and converters, nonlinear optics, optical amplifiers, optical fibers, parametric

\section{Introduction}

Parametric amplification in optical fibers has been initially studied in the 1980s and then largely abandoned as a research field due to technological limitations. It wasn't until recently that major breakthroughs, including the development of highly nonlinear fibers and efficient Brillouin suppression techniques, have brought parametric amplification back on the research scene and positioned it as a leading technology for future all-optical ultrafast signal processing [1-6]. In addition to providing broadband and high gain in many spectral bands, fiber optical parametric amplifiers (FOPAs) exhibit low noise figures [7], equivalent to those of conventional optical fiber amplifiers (e.g., erbium-doped fiber amplifiers (EDFAs), Raman amplifiers) or even better when they operate in a phasesensitive configuration [8]. They additionally offer the possibilities to achieve wavelength band conversion and, as recently demonstrated, many other applications such as ultrafast

Received 18 March 2008; accepted 25 April 2008.

*Arnaud Mussot is now with the Laboratoire de Physique des Lasers Atomes et Molécules, Université des Sciences et Technologies de Lille, 59655 Villeneuve d'Asq cedex, France.

$†$ Armand Vedadi is now with the Institute of Advanced Telecommunications, Swansea University, Singleton Park Swansea SA2 8PP Wales, United Kingdom.

LLaurent Provino is now with PERFOS, 11 rue de Broglie, 22300 Lannion, France.

Address correspondence to Thibaut Sylvestre, Département d'Optique P. M. Duffieux, Institut FEMTO-ST, CNRS UMR 6174, Université de Franche-Comté, 16 route de gray, 25030 Besançon, France. 
all-optical signal sampling, limiting buffering, high-repetition-rate pulse train generation, or time-division multiplexing [4-6].

Despite these advances and the vast number of papers published, however, it is significant that FOPA suffers from their complex and costly pumping scheme compared to conventional fiber amplifiers. Indeed, the pump generally consists of a tunable laser source, a high-speed phase modulator, and one or two high-power EDFAs in combination with bandpass filters. On one hand, the phase modulation necessary to suppress the stimulated Brillouin scattering (SBS) induces idler spectral broadening and signal distortion [9-13]. On the other hand, the remaining amplified spontaneous emission of optical amplifier used to reach continuous-wave $(\mathrm{CW})$ high pump power severely limits the noise figure [14].

In this article, we review the recent theoretical and experimental works performed at the FEMTO-ST Institute in Besançon, France. These works have been partially carried out under collaboration with the Alcatel Research \& Innovation Department in Marcoussis, France. After a brief recall of the principle of operation of FOPA, we will discuss the system performances of theses parametric devices such as gain bandwidth. Then we will focus on the main limitations caused by pump phase modulation. Finally, we will demonstrate an efficient technique for suppressing them using an integrated synchronized double phase modulator in a dual-pumped fiber optical parametric amplifier.

\section{Principle of Parametric Amplification}

The underlying nonlinear process for FOPA is the optical Kerr effect which causes a variation in index of refraction proportional to the local intensity of the light. When a high-power pump wave and a frequency-detuned small signal wave travel together in an optical fiber, they produce interference beats and modulate the refractive index via the Kerr effect, leading to the generation of a light-induced moving index grating. This phenomenological approach of parametric amplification is schematically sketched in Figure 1. In such a scheme, parametric amplification can be thought in the time domain as an energy transfer from the pump to both the signal and the idler waves by

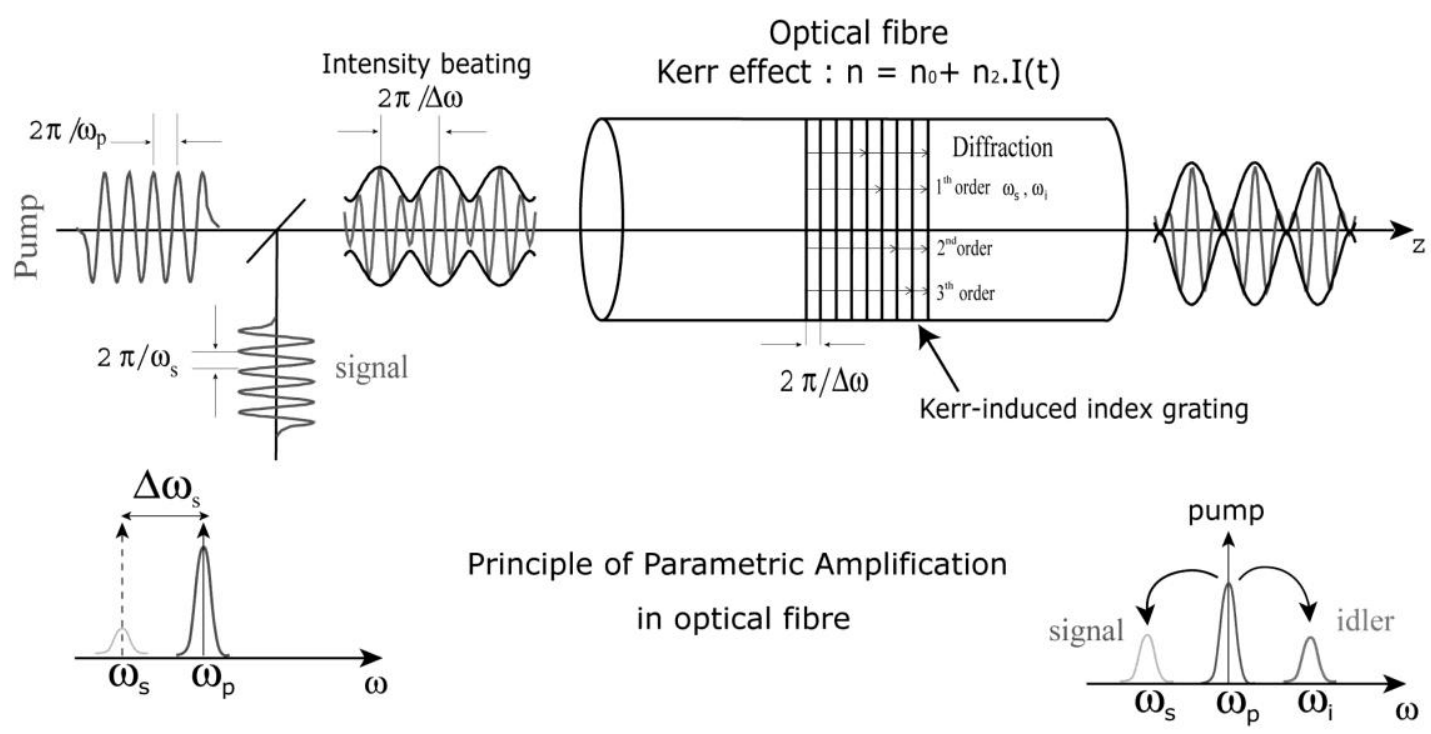

Figure 1. Principle of parametric amplification and phase-conjugated idler wave generation in optical fiber through the optical Kerr effect. 
a diffraction-like process on the refractive index grating [15]. In the frequency domain, parametric amplification can be viewed as a four-wave mixing (FWM) process where the pump and the signal are present at the fiber's input end. When phase matching between these two waves is fulfilled, exponential-like amplification of the signal together with generation of an idler wave is made possible. The phase matching conditions and the parametric gain can be related to the index grating period and the contrast, respectively. Phase matching is generally satisfied when nonlinear phase effects compensate for the linear ones. More precisely, this can be achieved in the anomalous dispersion regime of optical fibers where positive nonlinear phase modulation compensates for the negative group-velocity dispersion.

\section{Flat and Broadband Parametric Gain}

It is well known that in FOPA, the shape of the gain curve depends both on the input waves (pump, signal, and idler) and on the fiber characteristics, mainly its groupvelocity dispersion (GVD), nonlinear coefficient $\gamma$, and length $L[15,16]$. If the nonlinear coefficient $\gamma$ and the pump power $P_{0}$ are fixed, dispersion characteristics govern the shape of the small-signal gain curve while the magnitude of the gain depends on the fiber length. By tuning these two parameters, length and zero-dispersion wavelength $\lambda_{0}$, we can control the gain profile generated in optical fibers.

In this section, we describe a simple scheme to allow for the achievement of flat gain over ultrabroad bands with a single pump FOPA operating in the zero-dispersion wavelength region $[17,18]$. The proposed method, based on a multi-section dispersiontailored in-line nonlinear fiber arrangement, is depicted in Figure 2. The dispersion characteristics and lengths of the fiber sections are chosen in order that the first long piece generates the main broad gain profile and the following sections smooth the gain curve. Thus, it is possible to design a multi-section fiber with a particular management of the zero-dispersion wavelength to achieve a broadband and flat gain. The minimal number of fibers $N$, as well as their optimum lengths $L_{i}(i=1, \ldots, N)$, and their zero-dispersion wavelengths $\lambda_{0}^{i}$, are obtained by solving the nonlinear Schrödinger equation (NLSE), including the second-, third-, and fourth-order dispersion coefficients of each fiber.

To solve the NLSE, we used the numerical split-step Fourier method and the analytical FWM theory. On the whole of the obtained results, both methods are proved to be in excellent agreement. We also took into account in our simulation a power splicing loss of $0.6 \mathrm{~dB}$ between two fiber segments. We found that a broadband and flat gain bandwidth can be obtained using four fiber segments $(N=4)$, which is the minimum number required to reach such a feature. The multi-section amplifier consists in a set of highly nonlinear fibers (HNLFs) whose parameters can be found in [17]. The zero-dispersion wavelength can be tuned over a range of a few $\mathrm{nm}$ around the average value $\lambda_{0}=$ $1,550 \mathrm{~nm}$ according to the doping level and the core radius of the fibers. For all HNLF's,

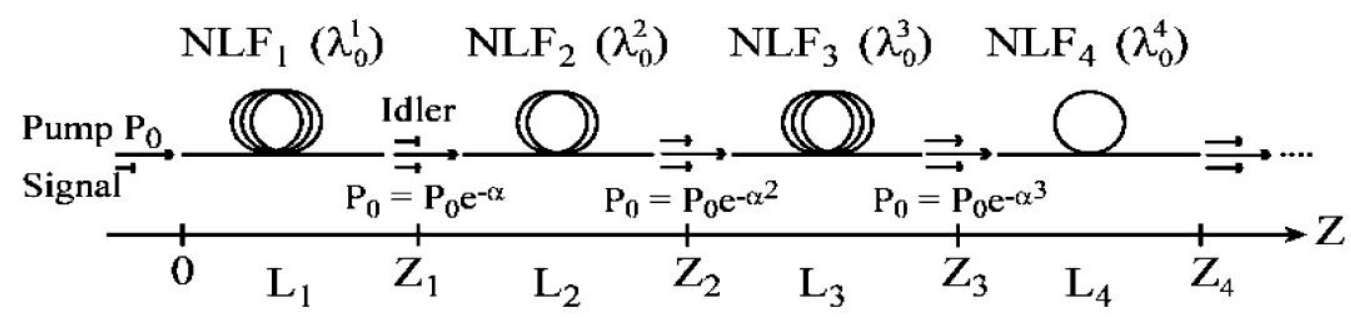

Figure 2. Schematic of the proposed multiple-fibers optical parametric amplifier architecture. 
the dispersion slope was $0.031 \mathrm{ps} / \mathrm{nm}^{2} / \mathrm{km}$, the nonlinear coefficient $\gamma=20 \mathrm{~km}^{-1} \mathrm{~W}^{-1}$, and the fourth-order dispersion $\beta_{4}$ was equal to $-2.85 \times 10^{-55} \mathrm{~s}^{4} \mathrm{~m}^{-1}$, which is a realistic value in HNLF.

Figure $3 \mathrm{a}$ shows a typical example of our theoretical results. A nearly flat parametric gain with a broad bandwidth of 100-nm is achieved for a total amplifier length of $422 \mathrm{~m}$ ( $\lambda_{s}$ denoted the signal wavelength). The pump power is $P_{0}=500 \mathrm{~mW}$ and the pump wavelength is $\lambda_{P}=1,550 \mathrm{~nm}$. Peak-to-peak gain uniformity is of $0.2 \mathrm{~dB}$ over $70 \mathrm{~nm}$ with $11.8 \mathrm{~dB}$ maximum gain. Figure $3 \mathrm{a}$ also shows the parametric gain obtained after each section of HNLF that illustrates the progressive compensation of the parametric gain oscillations. The optimum values of $L_{i}$ and $\lambda_{0}^{i}$ are chosen so that the maximum gain in a considered section corresponds to the minimum gain in the previous section. Figure $3 \mathrm{~b}$ depicts the evolution of the small-signal gain profile during propagation along the amplification line. It is important to emphasize that this gain flattening technique has been partially validated by recent experimental measurements [19]. In addition, the

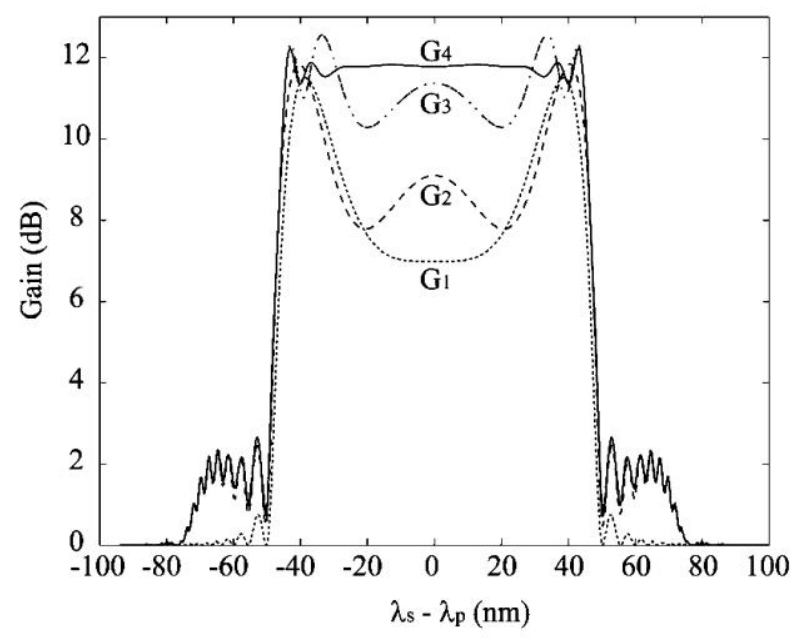

(a)

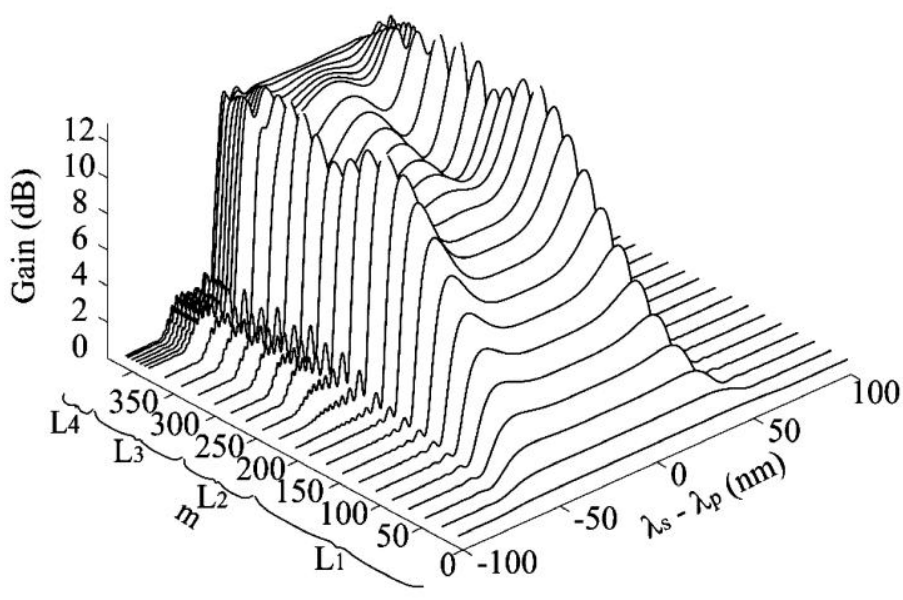

(b)

Figure 3. (a) Small-signal gain versus wavelength in a multiple-fibers parametric amplifier for a pump power $P_{0}=500 \mathrm{~mW}$ (solid line). Curves $G_{1}$ to $G_{4}$ show the successive output gain spectra calculted after each fiber section. (b) Numerical simulation of the gain spectrum versus propagation distance. 
robustness of this gain-flattening technique has been also numerically checked against random fluctuations of the zero-dispersion wavelength in each of the fiber sections [17]. Indeed, a single-pump FOPA is strongly sensitive to longitudinal fluctuations of the zerodispersion wavelength because they impair the phase matching conditions. Note that this dependence can be advantageously used to map the zero-dispersion wavelength along an optical fiber, as recently demonstrated using parametric gain measurements for different pump wavelengths [20].

\section{Impact of Pump Phase Modulation}

One of the major advantages of FOPA-based devices is that they can be fully transparent and independent of the data modulation format and bit-rate. This independence arises because the underlying FWM mechanism relies on the quasi-instantaneous optical Kerr effect and thereby retains both the amplitude and the phase of original signal. This unique property is valid under the assumption of an ideal monochromatic CW pump. For practical considerations, however, FOPAs are, in most cases, driven by phase-modulated CW pump schemes to avoid both synchronization problems and backward SBS.

In this section, we revisit, analytically and numerically, the principle of operation of FOPA by taking into account the phase modulation of the pump wave [9]. We show that it can induce strong gain modulation that interferes with the data modulation format. In particular, our analytical results reveal that both the rise time of the modulator and the dispersion slope of the fiber are the main limiting parameters. We further illustrate the detrimental impact of these time-dependent parametric gain variations on a $10 \mathrm{Gbit} / \mathrm{s}$ signal by means of a relevant numerical example using a Pseudo-RandomBinary-Sequence (PRBS) for the phase modulation of the pump. Let us first consider the well-known nonlinear Schrödinger (NLS) equation that governs parametric amplification in optical fibers. Neglecting the fiber loss and including the higher-order dispersion terms, this NLS equation takes the following form [17]:

$$
j \frac{\partial A}{\partial z}=-\sum_{m=2}^{4} j^{m} \frac{\beta_{m}}{m !} \frac{\partial A^{m}}{\partial \tau^{m}}-\gamma|A|^{2} A
$$

where $A(\tau, z)$ is the slowing varying amplitude of the electrical field, $\tau=t-z / v_{g}$ the time expressed in a reference frame moving with the group velocity of the pump wave, $\beta_{m}$ the $m$ th dispersion order and $\gamma$ the nonlinear coefficient. Equation (1) is known to possess a steady-state $\mathrm{CW}$ solution and parametric amplification of a small signal is purposely examined by a linear stability analysis of its CW solution,

$$
A(\tau, z)=\left(\sqrt{P_{0}}+u(\tau, z)\right) \times \exp \left[j\left(\gamma P_{0} z+\varphi(t)\right)\right]
$$

where $P_{0}$ is the pump power, $u(\tau, z)$ the small signal and $\varphi(t)$ a time-dependent term that accounts for the pump phase modulation. Linearizing with respect to $u$ and keeping only the first derivative of the pump phase, one gets an expression that governs the small signal amplification (see [9]). Finally, the parametric gain can be expressed as:

$$
g^{2}(\tau)=-\left(\Delta \beta_{L}+\delta \beta_{P M}(t)\right)\left(\gamma P_{0}+\frac{\Delta \beta_{L}+\delta \beta_{P M}(t)}{4}\right),
$$


where $\Delta \beta_{L}=\beta_{2} \Delta \omega^{2}+\frac{\beta_{4} \Delta \omega^{4}}{12}$ is the usual linear phase mismatch, $\Delta \omega$ is the pump-signal frequency detuning, and

$$
\delta \beta_{P M}(\tau)=\beta_{2} \varphi_{\tau}^{2}-\beta_{3} \varphi_{\tau} \Delta \omega^{2}+\frac{\beta^{4}}{12}\left[\varphi_{\tau}^{4}+4 \varphi_{\tau}^{3} \Delta \omega+6 \varphi_{\tau}^{2} \Delta \omega^{2}\right]
$$

is the instantaneous phase mismatch induced by the pump phase modulation. For a typical broadband FOPA, one has $\varphi_{\tau}=\partial \varphi / \partial \tau \ll \Delta \omega$ and one can approximate Eq. (3) by a simpler expression as

$$
g^{2}(\tau)=-\left(\frac{\beta_{4} \Delta \omega^{4}}{12}-\beta_{3} \Delta \omega^{2} \varphi_{\tau}\right)\left(\gamma P_{0}+\frac{\beta_{4} \Delta \omega^{4}}{48}-\frac{\beta_{3} \Delta \omega^{2}}{4} \varphi_{\tau}\right) .
$$

Equations (3) and (5) show that the parametric gain becomes time-dependent and depends both on the derivative of the pump phase and the fiber dispersion slope. Note that if we set $\varphi_{\tau}=0$ in Eqs. (3) and (5), we can readily find the usual expression of the parametric gain for the case of a monochromatic pump [3,4]. Figures $4 a-4 d$ illustrate the influence of pump phase modulation on the parametric gain of a $\mathrm{CW}$ signal. We use the typical parameters of a FOPA based on a HNLF. Additionally, we assume a $3 \mathrm{GHz}$ PRBS phase modulation of the pump with 27 ps rise/fall time of the modulator. In Figure 4a we depict
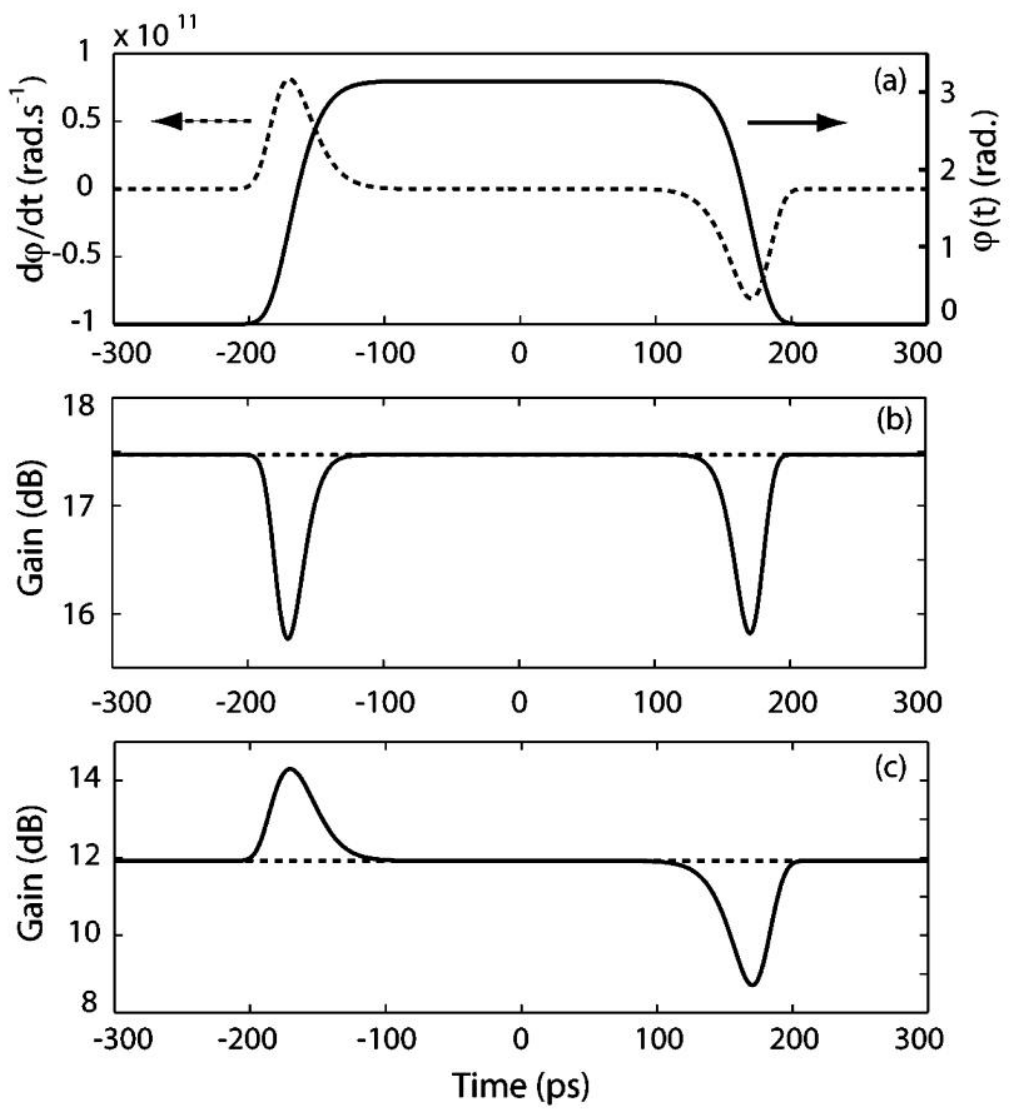

Figure 4. (a) Phase of the pump wave (solid line, right scale) and its associated time derivative (dashed line, left scale). (b) Instantaneous parametric gain of a CW signal detuned by $4.7 \mathrm{THz}$ from the pump (solid line) and usual parametric gain (dashed line); (c) same as (b) but with a $3 \mathrm{THz}$ detuning. 


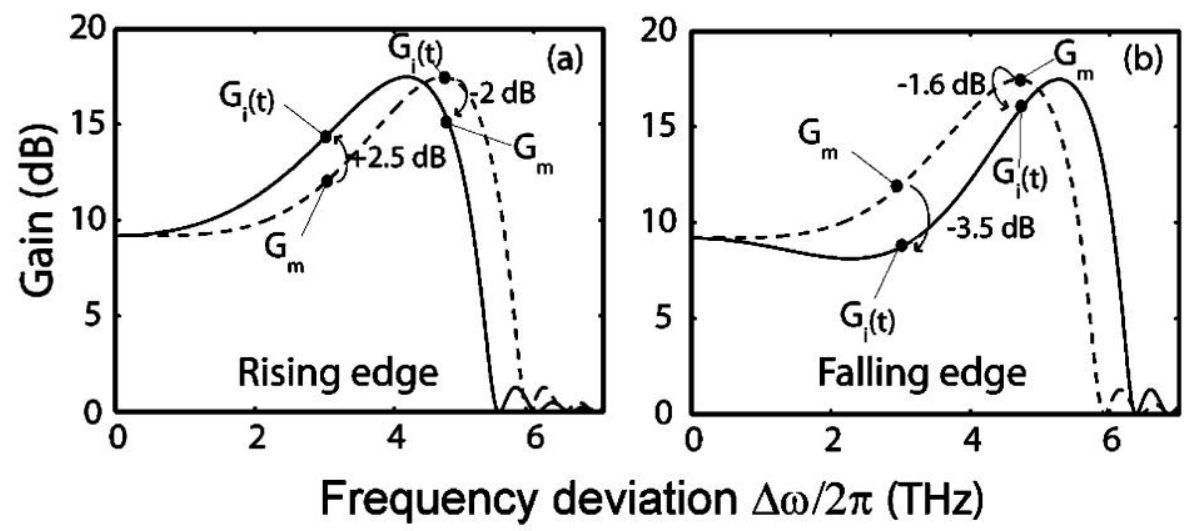

Figure 5. Parametric gain bands for (a) a rising and (b) a falling edge of the pump phase (solid lines), and for $\partial \varphi / \varphi \tau=0$ (dashed lines).

one bit of the phase sequence (solid line) and its associated time derivative (dashed line). Figures $4 \mathrm{~b}$ and $4 \mathrm{c}$ show the instantaneous parametric gain in decibels, calculated from Eq. (3), for two small signals. The first one is located at the maximum of the parametric gain curve $(4.7 \mathrm{THz}$, see Figure 5) and the other near the inflection point of this curve ( $3 \mathrm{THz}$ ). As it can be easily seen, the phase modulation of the pump has a detrimental impact on the temporal evolution of the parametric gain during both the leading and falling edges of the pump phase. Indeed, the gain suffers from sharp distortions, either positive or negative, compared to the constant parametric gain for a monochromatic pump (see dashed lines in Figures $1 \mathrm{~b}$ and 1c). The physical interpretation of these phase-induced gain distortions is quite intuitive. The phase modulation of the pump leads to an instantaneous frequency chirp that will modify the phase matching condition. As a consequence, the parametric gain differs from its nominal value during each phase jump of the pump wave. To gain better insight, we have plotted in Figures $5 \mathrm{a}$ and $5 \mathrm{~b}$ the instantaneous gain curves (solid lines) for the two extreme values of the phase derivative which correspond to the rising and falling edges of pump phase presented in Figure $4 \mathrm{a}$, respectively. As a comparison, the dashed curve of Figures $5 \mathrm{a}$ and $5 \mathrm{~b}$ shows the parametric gain for a monochromatic pump. For the signal shifted by $3 \mathrm{THz}$ from the pump, the nominal gain is $G_{m}=12 \mathrm{~dB}$ and reaches $G_{i}=14.5 \mathrm{~dB}$ when $\partial \varphi / \partial \tau=1.10^{-11} \mathrm{rad} \cdot \mathrm{s}^{-1}$, in good agreement with the peak gain modulation in Figure $4 \mathrm{c}$. The same explanation stands for the falling edge of the pump phase (Figure $5 b$ ), that induces a decrease of $-3.5 \mathrm{~dB}$ in the gain, and can be applied to all frequencies included in the gain band.

\section{Bit-Error Rate Measurements}

To quantify the signal gain distortions of a parametrically amplified channel, bit-error rate (BER) measurements were carried out at the output of a FOPA [10]. The experiment was performed in the Alcatel Research and Innovation department, in Marcoussis, France. The FOPA under test is depicted in Figure 6. The pump at wavelength $\left(\lambda_{p}=1,553.3 \mathrm{~nm}\right.$ is phase-modulated by a $1.5 \mathrm{Gbit} / \mathrm{s}$ PRBS, to increase the SBS threshold. Then it is amplified by two EDFAs with a filtering of the amplified spontaneous emission (ASE) in between. The pump is finally injected in the fiber through a 90/10 coupler, yielding to a pump power of $330 \mathrm{~mW}$. The amplifying fiber used in this FOPA is a highly nonlinear dispersionshifted fiber (HNLF, $L=490 \mathrm{~m}$ and $\left(\lambda_{0}=1,553 \mathrm{~nm}\right.$ ). The total fiber losses (fiber attenuation + splicing losses) are $0.8 \mathrm{~dB}$. On the signal path, a polarization controller 


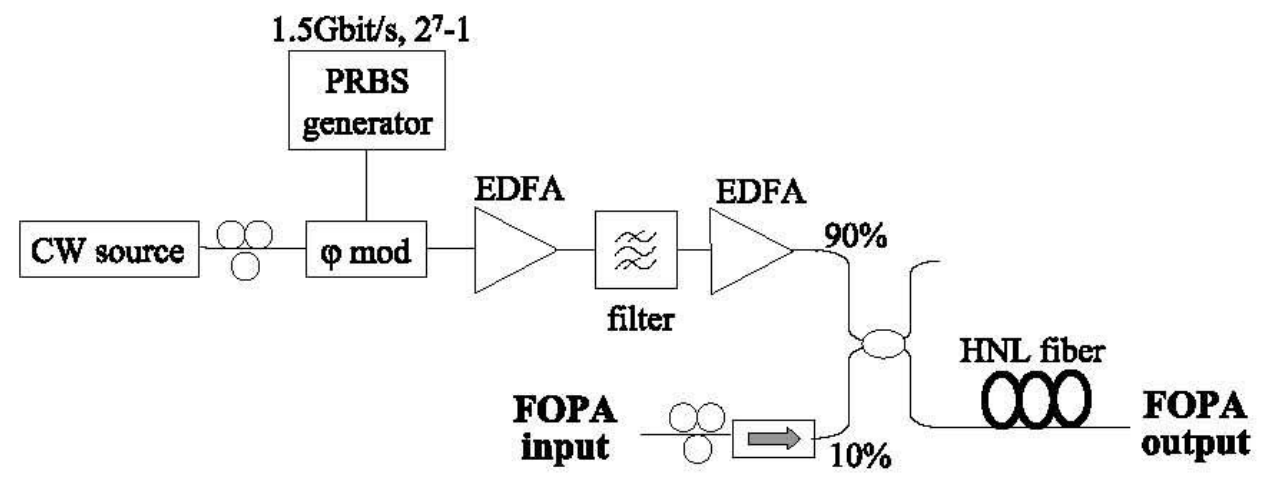

Figure 6. Experimental set-up of our fiber optical parametric amplifier under test.

allows us to adjust the signal polarization to maximize the gain and an isolator protects the input from back reflections.

BER measurements were performed on a $10 \mathrm{Gbit} / \mathrm{s}$ non return to zero (NRZ) modulated channel. The experimental set-up is sketched in Figure 7. At the output of the FOPA, a circulator and a fiber Bragg grating, similar to those used in the FOPA, allows for removing the residual pump. The receiver is made up of an $L$-band preamplifier and a filter with a $3 \mathrm{~dB}$-bandwidth of $0.23 \mathrm{~nm}$ to reduce the ASE-signal beat noise. BER is then plotted versus the optical signal to noise ratio (OSNR), which is measured with the optical spectrum analyzer OSA2, and for a constant optical power $(-11.3 \mathrm{dBm})$ on the photodiode. By means of a variable attenuator $A t t_{1}$ placed before the pre-amplifier, the OSNR can be decreased in front of the receiver to achieve measurable BERs. Back-toback measurements were carried out by connecting the point $\mathrm{A}$ to the point B. Finally, the on/off parametric gain of the FOPA is measured by using OSA1.

BERs were first measured in a back-to-back configuration, then with the FOPA by applying three different modulation formats on the pump phase [4]. First a PRBS at $3.5 \mathrm{Gbit} / \mathrm{s}$ was used, with a word length of $2^{7}-1$, which modulates the pump phase between 0 and $\pi$, then the same PRBS, filtered by an electrical filter with a $3 \mathrm{~dB}$ bandwidth of $3.04 \mathrm{GHz}$ and finally a combination of 4 sinusoidal signals $\left(f_{1}=120 \mathrm{MHz}\right.$, $f_{2}=532 \mathrm{MHz}, f_{3}=942 \mathrm{MHz}$, and $f_{4}=1.81 \mathrm{GHz}$ ). The electrical filtering of the PRBS enables to increase the rise-time of the phase bits from 100 to $150 \mathrm{ps}$. In the case of the sinusoid modulation format, a rather low Brillouin threshold of $300 \mathrm{~mW}$ was achieved. In order to compare the impact of the different modulation formats, BERs were measured in the same pumping conditions $\left(P_{p}=280 \mathrm{~mW}\right)$ and for the same channel

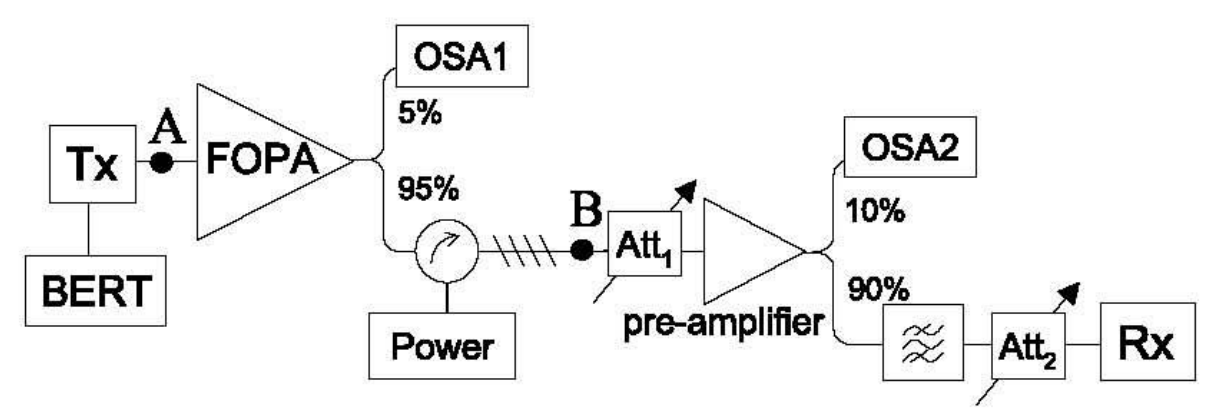

Figure 7. Experimental set-up for the BER measurements. 


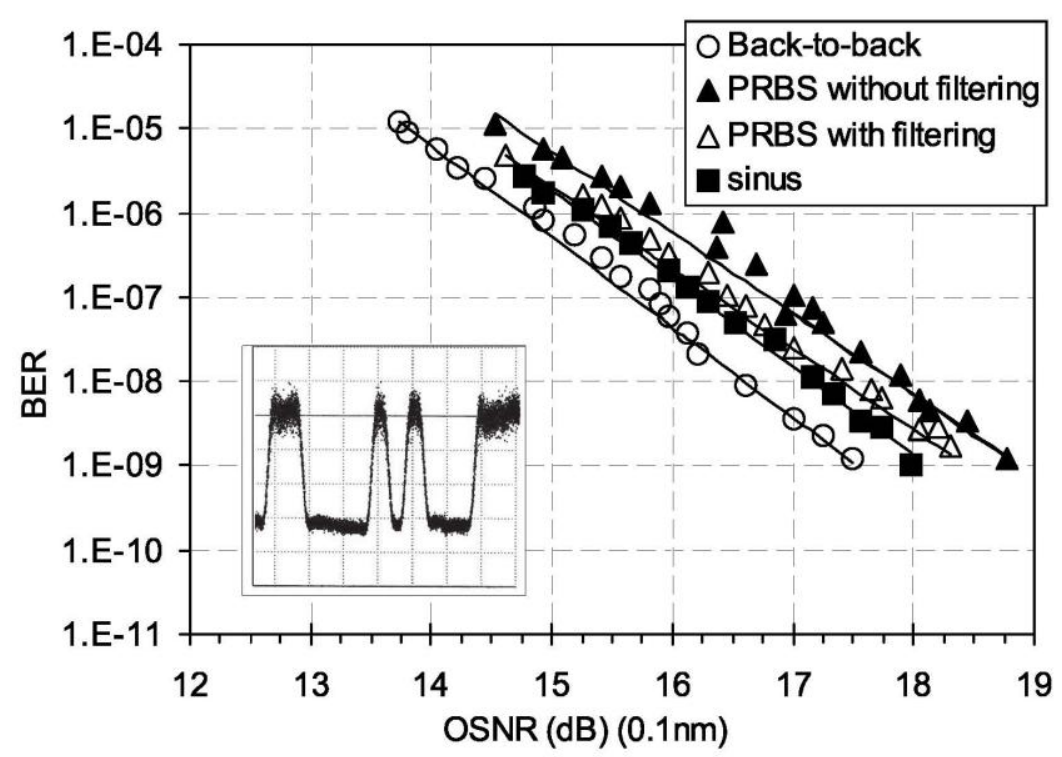

Figure 8. OSNR penalty induced by 3 different modulation formats of the pump phase, $P_{p}=$ $280 \mathrm{~mW}, \lambda_{\text {channel }}=1,595 \mathrm{~nm}$. Inset: bits of the amplified channel measured with an oscilloscope.

wavelength at $1,595 \mathrm{~nm}$ (about $42 \mathrm{~nm}$ from the pump), which corresponds to a maximum On/Off gain of $6.7 \mathrm{~dB}$.

The results of BER measurements are presented in Figure 8. The impact of the pump phase modulation on the performance of the FOPA can be clearly seen in terms of OSNR penalty. Actually, the OSNR was increased to achieve the same BER for the three different modulation formats as in the back-to-back configuration. The OSNR penalty for a bit error rate of $10^{-9}$ is $1.38 \mathrm{~dB}$ for the PRBS, $0.91 \mathrm{~dB}$ for the filtered PRBS, and $0.59 \mathrm{~dB}$ for the combination of sinusoids. First, the increase of the rise-time of the PRBS phase bits with the electrical filter leads to a reduction of the penalty by $0.47 \mathrm{~dB}$. Second, in the case of the combination of four sinusoids, the pump phase experiences more continuous variations than the PRBS cases. The change of the phase-matching condition is not as significant as in the PRBS cases, and thus the penalty is further reduced by $0.32 \mathrm{~dB}$.

As all measurements were performed in the same operating conditions except the phase modulation formats, these experiments clearly highlights the signal gain distortions induced by the pump phase modulation. Moreover, in the inset of Figure 8, it can be noticed that the mark level of the amplified channel is more distorted than the zero level, as predicted by the analytical results shown in Figure 4 . Nevertheless, it has recently been shown that, although the Q-value of data amplified by a periodic and deterministic gain may be considerably degraded, the BER can still be relatively unaffected if ON-OFF keyed (OOK) data is amplified [12].

\section{Demonstration of an Integrated $\mathrm{LiNbO}_{3}$ Synchronized Double Phase Modulator}

To overcome the impact of pump phase modulation, several techniques have been proposed [21-26]. Idler spectral broadening has been cancelled by using a dual-wavelength pumping counter phase modulation scheme $[22,23]$. The counter phase modulation was employed so that the idler frequency chirp (or equivalently the signal gain distortion) induced by one pump is exactly balanced by an opposite frequency chirp induced by 
the second pump. This has been achieved either by using two-phase modulators (PMs) driven with two complementary patterns, or a single PM and an accurate optical delay line between the two pumps [24]. In both cases however, it is significant that the pumps' synchronization is hard to satisfy because of the short PM rise/fall time ( $\approx 30 \mathrm{ps}$ ).

In this section, we report the fabrication of a novel $\mathrm{LiNbO}_{3}$-based synchronized double phase modulator (SDPM) that allows for the simultaneous achievement of highspeed counter-phase modulation, synchronization, and coupling of two channels [27]. The effectiveness of this SDPM is clearly demonstrated in a broadband 2-pump FOPA through high-resolution spectral analysis of the idler and signal waves.

Our electro-optic phase modulator was designed and fabricated by Photline Technologies to meet the requirements of transparent FOPA applications. It consists of a synchronized double broadband modulator integrated on $\mathrm{X}$-cut $\mathrm{LiNbO}_{3}$. Figure $9 \mathrm{a}$ is a sketch of the device. It is composed of two input waveguides, linked by a Y-junction to a single output. All the waveguides are single-mode in the $1.55 \mu \mathrm{m}$ telecom window. A set of push-pull broadband co-planar RF-electrodes is put on top of both parallel waveguides. Thanks to a precise geometrical arrangement, they provide an RF-electric field of opposite sign in the optical arms. As an external electric signal is applied, each waveguide is the location of an index modulation that is the exact opposite, in amplitude and phase, of the modulation that takes place in the second waveguide.

A Y-coupler is set at the output for optical mixing. The device acts as two automatically synchronized and coupled broadband counter-phase PMs. It is fully packaged for RF-application up to $40 \mathrm{GHz}$. The eye diagram shown in Figure $9 \mathrm{~b}$ is the intensity modulation detected at the output of the device when launching the same monochromatic source at both inputs. In that configuration, the SDPM behaves as a Mach-Zehnder interferometer. We use here a narrow linewidth distributed feedback (DFB) fiber laser at $1,549.74 \mathrm{~nm}$. Figure $9 \mathrm{~b}$ shows the clearly opened eye diagram when driven by a $1 \mathrm{Gbits} / \mathrm{s}$ PRBS $2^{7}-1$ NRZ pattern.

The experimental set-up of our 2-pump FOPA that uses the SDPM is depicted in Figure 10. Two tunable lasers (TL1 and TL2) at $\lambda_{1}=1,536 \mathrm{~nm}$ and $\lambda_{2}=1,566 \mathrm{~nm}$ are co-polarized and then coupled into the SDPM. It is electrically driven by a PRBS pattern generator at $3 \mathrm{Gbits} / \mathrm{s}$ with a $2^{15}-1$ pattern (rise time of $30 \mathrm{ps}$ ). At the SDPM's output, the synchronized counter-phase modulated TLs are amplified using a single $33 \mathrm{dBm}$ high-power EDFA. A dual-channel fiber Bragg grating centered at $\lambda_{1}$ and $\lambda_{2}$ is used to remove any ASE noise in the amplification band. This custom filter was manufactured by Redfern Optical Components and consists of two sub-gratings written in a single-
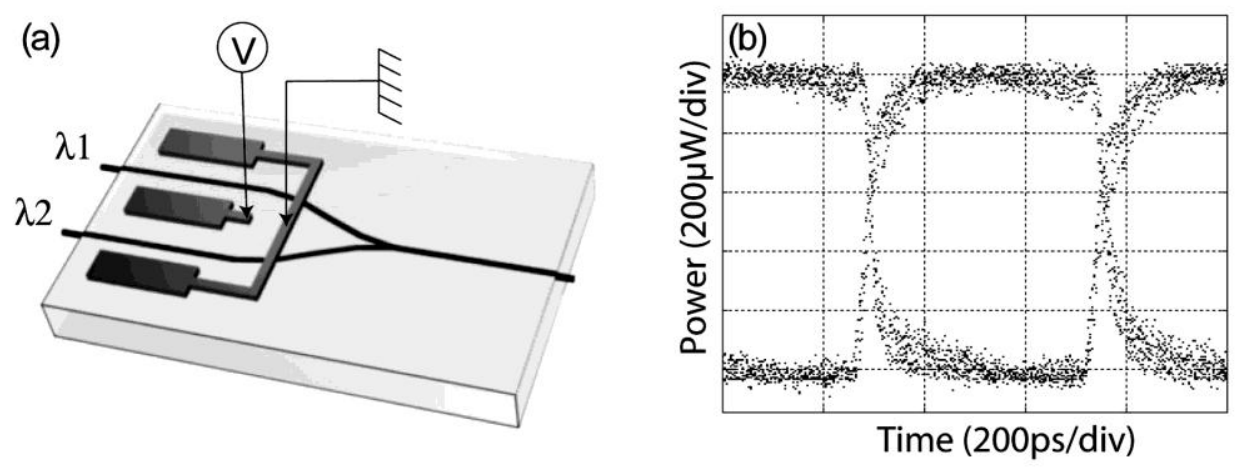

Figure 9. (a) Scheme of the X-cut synchronized double phase modulator. (b) 1 Gbits/s NRZ-eye diagram. 


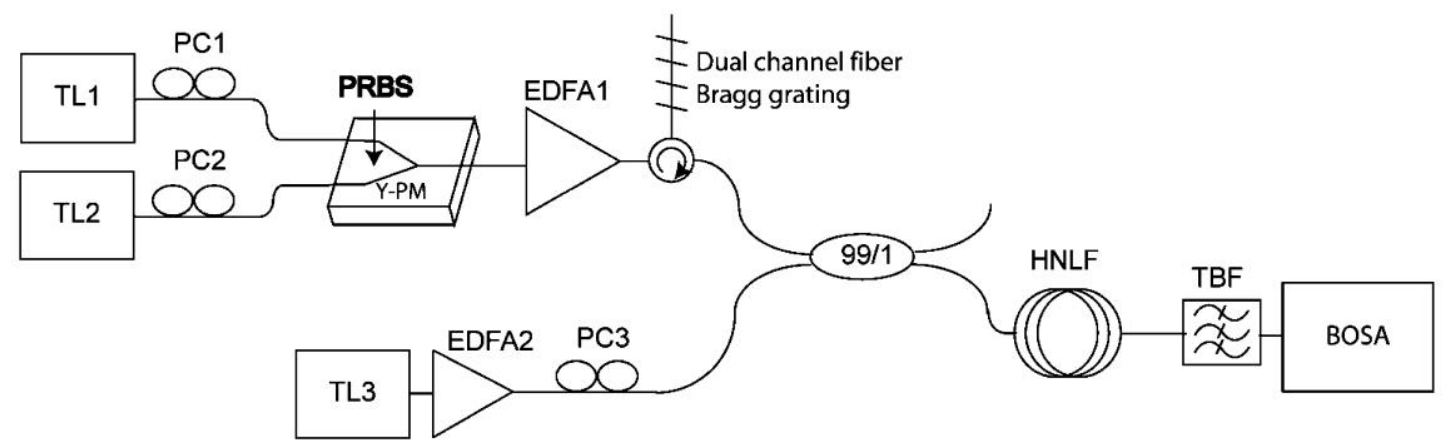

Figure 10. Experimental set-up of the counter-phase modulated two-pump fiber optical parametric amplifier. TL: tunable laser; PC: Polarization controller; PRBS: Pseudo-random bit sequence; TBF: Tunable bandpass filter; HNLF: Highly nonlinear fiber; BOSA: Brillouin optical spectrum analyzer.

piece of fiber at the same location, thus keeping the pumps in synchronization. As a signal source, we use another CW TL3. It is slightly amplified and launched together with the pumps into the amplifying fiber through a 99/1 coupler. The signal power and wavelength are $-16 \mathrm{dBm}$ and $\lambda_{S}=1,547 \mathrm{~nm}$, respectively. The amplifier medium is a $500 \mathrm{~m}$-long highly nonlinear fiber with nonlinear coefficient $\lambda=11 \mathrm{~W}^{-1} \mathrm{~km}^{-1}$ and zero-dispersion $\lambda_{0}=1,551.4 \mathrm{~nm}$. The $3 \mathrm{rd}$ and 4 th dispersion coefficients were measured using a recent method [28]. TL1 and TL2 powers are tuned to reach $23 \mathrm{dBm}$ on each pump. At the output end, the amplified signal and idler waves are independently analyzed using a tunable bandpass filter (TBF, 1-nm bandwidth) and a Brillouin optical spectrum analyzer (BOSA) with a 10-MHz high-resolution. The counter-phase modulation scheme is finally compared to the in-phase scheme simply by launching TLs together in one input waveguide of the SDPM using a fiber coupler.

Figure 11 illustrates the experimental FOPA spectrum with the two pumps, the amplified signal and the generated idler at $\lambda_{I}=1,555 \mathrm{~nm}$. We measured a $10 \mathrm{~dB}$ on/off gain, in good agreement with the theoretically predicted gain plotted in dashed line in Figure 11 [26]. Figures 12 and 12c show the signal and idler spectra when the pumps are in-phase modulated. In particular, Figure 12c clearly shows the deterioration and the

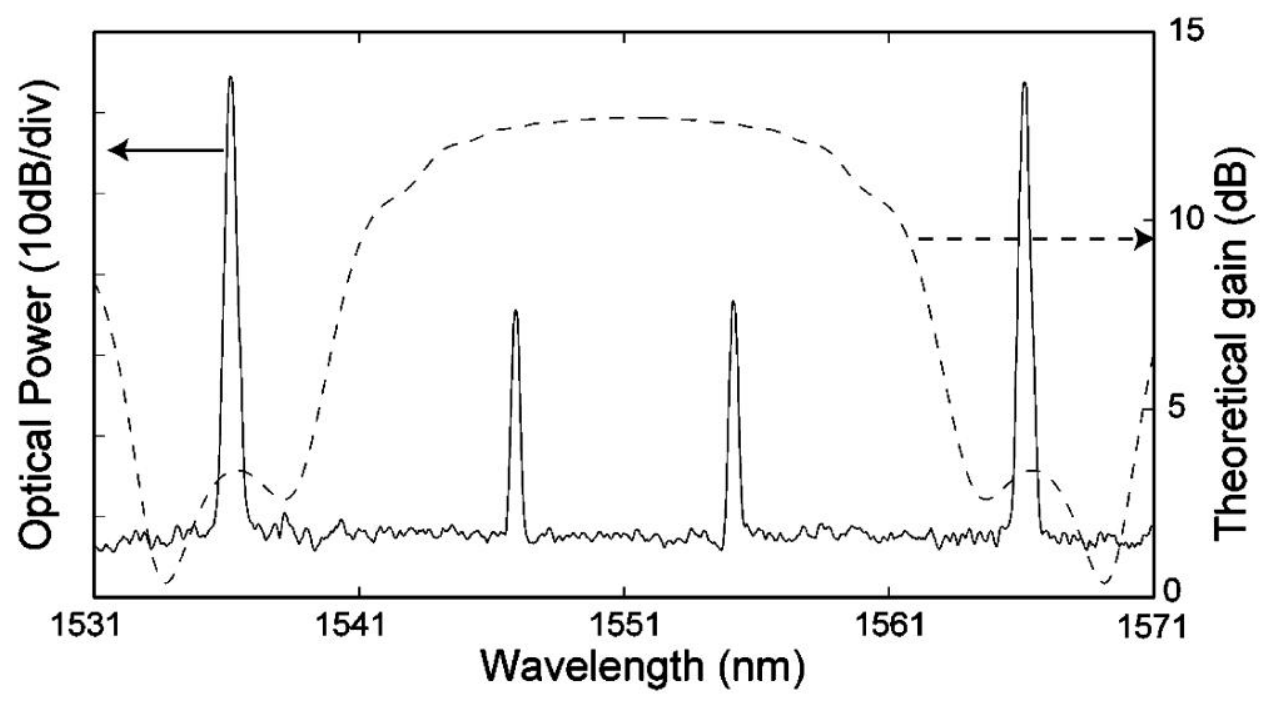

Figure 11. Experimental output spectrum of the FOPA (solid line) and theoretical FOPA gain bandwidth (dashed line). 


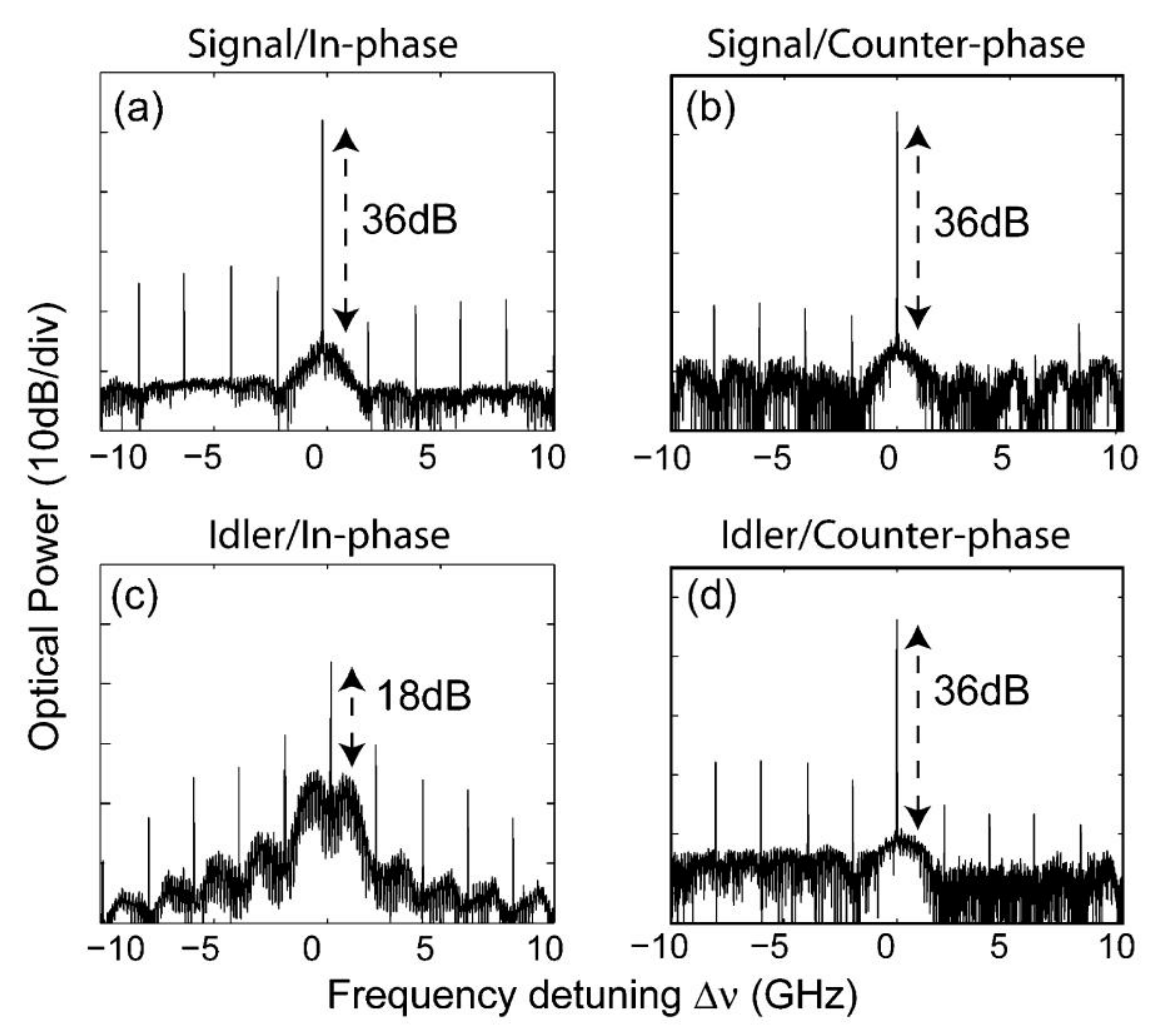

Figure 12. (a), (c) Signal and idler spectra when the pumps are in-phase modulated and (b), (d), counter-phase modulated with the SDPM.

spectral broadening of the idler with respect to the output signal. The idler peak power is at least $10 \mathrm{~dB}$ lower while the ratio to the pedestal noise is $17 \mathrm{~dB}$ lower compared to the output signal.

In the counter-phase modulation scheme, however, we can see on Figures $12 \mathrm{~b}$ and $12 \mathrm{~d}$ that the idler broadening is totally cancelled and the ratio of the idler to the pedestal noise is comparable to that of the output signal. These experimental results clearly show the effectiveness of our double phase modulator to suppress the impairment of pump phase modulation on the system performances of FOPA.

\section{Conclusion}

In this article, we presented some of the recent research works about fiber-based parametric amplifiers and their system performances done in the Optics Department of the FEMTO-ST Institute at the University of Franche-Comté in Besançon, France. We have theoretically investigated a multi-section fiber-optical parametric amplifier that provides a nearly flat gain over a 100-nm bandwidth without any gain-equalization filter. Then, we have revisited theoretically the principle of operation of parametric amplifiers by taking into account the phase modulation of the pump wave, necessary in practice to avoid stimulated Brillouin scattering. We have shown that the rise/fall time of the modulator can induce distortions in the parametric gain and, in particular, that the fiber's dispersion slope plays an important role. The OSNR penalty of a fiber optical parametric amplifier has been quantified through BER measurements with a $10 \mathrm{Gbit} / \mathrm{s}$ line rate performed at Alcatel in Marcoussis, France. By comparing three-phase modulation formats, it has been shown that, beyond the efficiency of the pump phase modulation to suppress the Brillouin 
scattering, attention should be paid to the associated extra penalty experienced by the channels. The lowest penalty of $0.59 \mathrm{~dB}$ was achieved with a phase modulation format including four sinusoids. The influence of the pump phase modulation was also evidenced by the distortion of the mark level of the amplified channel. The current development of nonlinear fibers and photonic crystal fibers with ultra-low dispersion slopes would be potentially beneficial in order to overcome this impairment in single pump FOPA [29]. We have additionally demonstrated a novel two-pump fiber optical parametric amplifier architecture including a single $\mathrm{LiNbO}_{3}$-based synchronized double phase modulator for suppressing this impairment. With this phase modulator, we achieved counter-phase modulation of the pumps and demonstrated idler spectral broadening suppression, paving the way for fully transparent parametric devices. Our results highlight the crucial issue of pump wave characteristics on the performances of parametric amplifiers. While one pump scheme allows for the achievement of efficient signal-processing all-optical techniques, counter-phase modulation of two low-noise pumps seems to be the best solution since it can suppress both idler spectral broadening and signal gain distortions. However, we must stress that these pumping scheme requires costly high-speed phase modulation schemes and high-power EDFAs. Moreover, the noise figure of parametric amplifiers is usually degraded by the remaining ASE from the EDFA. The use of the recently developed low-noise narrow-linewidth high-power fiber laser as a parametric pump may be an alternative cost-effective pumping scheme. Finally, it is now possible to think about alternative solutions for passive suppression of Brillouin scattering that require no pump linewidth broadening, by using for instance the phononic bandgap properties of microstructured fibers $[29,30]$.

\section{Acknowledgments}

This work was partially supported by Alcatel Research and Innovation and by the Conseil Régional de Franche-Comté. The authors thank A. Durecu-Legrand, C. Simmoneau, and D. Bayart for fruitful collaboration, N. Grossard and J. Hauden from Photlines Technologies for providing the optical phase modulator, and F. Patois and J. M. Merolla for loaning the BOSA. The authors also thank Sumitomo Electrics for providing the highly nonlinear fibers.

\section{References}

1. Stolen, R. H., and Bjorkholm, J. E. (1982). Parametric amplification and frequency conversion in optical fibers. IEEE J. Quantum Electron. 18:1062-1072.

2. Pocholle, J. P., Raffy, J., Papuchon, M., and Desurvire, E. 1985. Raman and four photon mixing amplification in single mode fibers. Opt. Eng. 24:600-610.

3. Marhic, M. E. 2007. Fiber Optical Parametric Amplifiers, Oscillators and Related Devices. Cambridge, UK: Cambridge University Press.

4. Hansryd, J., Andrekson, P. A., Westlund, M., Lie, J., and Hedekvist, P.-O. 2002. Fíber-based optical parametric amplifiers and their applications. IEEE. J. of Select. Topics. in Quantum Electron. 8:506-520.

5. McKinstrie, C. J., Radic, S., and Gnauck, A. H. 2007. All-optical signal processing by fiberbased parametric devices. Opt. and Photon. News 18(3):35-40.

6. Islam, M. N., and Boyraz, O.2002. Fiber parametric amplifiers for wavelength band conversion. IEEE J. of Select. Topics in Quantum Electron. 8:527-537. 
7. Blows, J. L., and French, S. E. 2002. Low-noise-figure optical parametric amplifier with a continuous wave frequency modulated pump. Opt. Lett. 27:491-493.

8. Imajuku, W., Takada, A., and Yamabayashi, Y. 2000. In-line coherent optical amplifier with noise figure lower than $3 \mathrm{~dB}$ quantum limit. Electron. Lett. 36:63-65.

9. Mussot, A., Durécu-Legrand, A., Lantz, E., Simmoneau, C., Bayart, D., Maillotte, H., and Sylvestre, T. 2004. Impact of pump phase modulation on the gain of fiber parametric amplifier. IEEE Photon. Technol. Lett. 16:1289-1291.

10. Durécu-Legrand, A., Mussot, A., Simmoneau, C., Bayart, D., Sylvestre, T., Lantz, E., and Maillotte, H. 2005. Impact of pump phase modulation on system performances of fiber optical parametric amplifiers. Electron. Lett. 41:83-84.

11. Kylemark, P., Karlsson, M., and Andrekson, P. A. 2006. Impact of phase modulation and filter characteristics on dual-pumped fiber-optical parametric amplification. IEEE Photon. Technol. Lett. 18:439-441.

12. Kylemark, P., Ren, J., Myslivets, Y., Alic, N., Radic, S., Andrekson, P. A., and Karlsson, M. 2007. Impact of pump phase-modulation on the bit-error rate in fiber-optical parametricamplifier-based systems. IEEE Photon. Technol. Lett. 19:79-81.

13. Chavez Boggio, J. M., Guimaraes, A., Callegari, F. A., Marconi, J. D., and Fragnito, H. L. 2005. Q penalties due to pump phase modulation and pump RIN in fiber optic parametric amplifiers with non-uniform dispersion. Opt. Comm. 249:451-472.

14. Durécu-Legrand, A., Simmoneau, C., Bayart, D., Mussot, A., Sylvestre, T., Lantz, E., and Maillotte, H. 2005. Impact of pump OSNR on noise figure for fiber optical parametric amplifiers. IEEE Photon. Technol. Lett. 17:1178-1180.

15. Gindre, D., Maillotte, H., Monneret, J., Lantz, E., and Froehly, C. 1994. Coherent picosecond parametric amplification through a Kerr-induced index grating in a single-mode fiber. Opt. Comm. 112:75-79.

16. Marhic, M. E., Kagi, N., Chiang, T. K., and Kazovsky, L. G., 1996. Broadband fiber optical parametric amplifiers. Opt. Lett. 21:573-575.

17. Inoue, K. Arrangement of fiber pieces for a wide wavelength conversion range by fiber fourwave mixing. Opt. Lett. 19:1189-1191.

18. Provino, L., Mussot, A., Lantz, E., Sylvestre, T., and Maillotte, H. 2003. Broadband and flat parametric amplifiers with a multi-section dispersion-tailored nonlinear fiber arrangement. J. Opt. Soc. of Am. B 20:1532-1537.

19. French, S. E., and Blows, J. L. 2001. Continuous wave optical parametric amplifier with flattened gain. In: Optical Amplifiers and Their Applications, Vol. 60 of OSA Trends in Optics and Photonics, N. E. Jolley, J. D. Minelly, and Y. Nakano (Eds.). Washington, DC: Optical Society of America. Postdeadline paper PD7.

20. Mussot, A., Lantz, E., Sylvestre, T., Maillotte, H., Durécu-Legrand, A., Simmoneau, C., and Bayart, D. 2006. Zero-dispersion wavelength mapping of a highly nonlinear fiber using parametric gain. IEEE Photon. Technol. Lett. 18:22-24.

21. Tanemura, T., Lim, H. C., and Kikuchi, K. 2004. Suppression of idler spectral broadening in highly efficient fiber four wave mixing by binary phase shift keying modulation of pump wave. IEEE Photon. Technol. Lett. 13:1328-1330.

22. Ho, M. C., Marhic, M. E., Wong, K. K. Y., and Kazovsky, L. G. 2002. Narrow-linewidth idler generation in fiber four-wave mixing and parametric amplification by dithering two pumps in opposition of phase. J. Lightwave Technol. 20:469-476.

23. Wong, K. K. Y., Marhic, M. E., and Kasovsky, L. G. 2003. Phase-conjugate pump dithering for high-quality idler generation in a fiber optical parametric amplifier. IEEE Photon. Technol. Lett. 15:33-35.

24. Radic, S., Jopson, R. M., Gnauck, A., McKinstrie, C. J., Centanni, J. C., and Chraplyvy, A. R. 2005. Stimulated Brillouin scattering suppression using a single modulator in twopump parametric architectures. Paper OWN5. Optical Fiber Communication Conference 2005, Anaheim, California, March 22-26. 
25. Jopson, R. J. Polarization-insensitive optical four-photon mixer with orthogonally-polarized pump signals. US Patent 5.386.314, 1995.

26. Vedadi, A., Mussot, A., Lantz, E., Maillotte, H., and Sylvestre, T. 2006. Theoretical study of gain distortions in dual-pump fiber optical parametric amplifiers. Opt. Comm. 267:244-252.

27. Vedadi, A., Beugnot, J.-C., Lantz, E., Maillotte, H., Hauden, J., and Sylvestre, T. 2007. Fiber optical parametric amplifier based on a novel synchronous double phase modulator. Paper OWQ2. Optical Fiber Communication Conference 2007, Anaheim, California, March 25-30.

28. Auguié, B., Mussot, A., Boucon, A., Lantz, E., and Sylvestre, T. 2006. Ultralow chromatic dispersion measurement of optical fibers with a tunable fiber laser. IEEE Photon. Technol. Lett. 18:1825-1827.

29. Russell, P. St. J. 2006. Photonic-crystal fibers. J. of Lightwave Technol. 24:4729-4749.

30. Laude, V., Khelif, A., Benchabane, S., Wilm, M., Sylvestre, T., Kibler, B., Mussot, A., Dudley, J. M., and Maillotte, H. 2005. Phononic band-gap guidance of acoustic modes in photonic crystal fibers. Phys. Rev. B 71:045107.

\section{Biographies}

Thibaut Sylvestre was born in Besançon, France, in 1973. He received the M.Sc degree and the Ph.D degree in "Sciences Pour l'Ingénieur" from the Université de Franche-Comté, Besançon, France, in 1995 and 1999, respectively. He then started a 2-year postdoctoral stay abroad in the field of nonlinear fiber optics at Optics and Acoustics Department of the Université libre de Bruxelles (ULB), Belgium. In 2001, he was appointed full-time researcher in charge at the Centre National de la Recherche Scientifique (CNRS) in the FEMTO-ST institute, Besançon, France. He is involved with theoretical and experimental studies of fundamental nonlinear optical phenomena with the aim of investigating potential applications to telecommunications and other domains. Thibaut Sylvestre is a member of The Optical Society of America.

Arnaud Mussot was born in Vesoul, France, on August 4, 1976. He received the Master degree in Electronics in 1998 and the Ph.D degree in Optical Engineering in 2004 from the Université de Franche-Comté, Besancon, France. His research interests were parametric amplification and supercontinuum generation in optical fibers. From 2004 to 2005 he was with the Commissariat à 1'Energie Atomique in Bordeaux, France, and worked on high energy Ytterbium lasers. Since 2005, he has been working as an associate professor at the Université des Sciences et Technologiques de Lille. He is mainly engaged in supercontinuum generation and in the study of instability processes in fiber ring cavities.

Armand A. Vedadi received the M.Sc degree from the Institute of National Telecommunications (INT) in Paris, France, on June 2004. From 2004 to 2007, he worked on the nonlinear optics team at the FEMTO-ST Institute at the Université de Franche-Comté in Besançon, France toward his Ph.D. Since October 2007, he has been working with the Institute of Advanced Telecommunications (IAT) at Swansea University, UK. His research interests focus on optical parametric amplification and nonlinear effects in optical fibers.

Laurent Provino was born in France on August 29, 1972. He received the Ph.D Degree in "Sciences Pour l'Ingénieur" from the Université de Franche-Comté, Besançon, France, in 2002. From 1998 to 2002, he worked on parametric amplification, optical fibers, microstructured optical fibers, and continuum generation for applications in future all-optical telecommunication systems. After two years of Post-Doctoral positions at the Institut Fresnel (University of Marseille) and University of Science and Technology of Lille, he joined PERFOS (Plate-forme d'Études et de Recherche sur les fibers Optiques 
Spéciales) in 2005, as an Optical Fiber Modelling Engineer. His current activities are simulations and design of microstructured optical fibers for various applications.

Eric Lantz was born in Paris, in 1957. From 1979 to 1985 , he worked in the field of solar energy and heat pumps. In 1986, he joined the Laboratoire d'Optique P. M. Duffieux of the Université de Franche-Comté and he received, in 1989, the Ph.D degree from the Université de Franche-Comté, where he studied super-resolution in optical microscopy by inverse problem method. In 2001, he was appointed Professor of Physics at the Département d'Optique in the Université de Franche-Comté. He is involved with studies of travelling-wave parametric image amplification in nonlinear crystals and its applications ranging from to time-gating to quantum imaging. His current research interests also include parametric amplification in optical fibers, spatial solitons, and optimal photon counting strategies with EMCCD cameras.

Hervé Maillotte was born in Besançon, France, in 1962. He received the Ph.D degree in Engineering Science in 1990 from the Université de Franche-Comté, Besançon, France. After about two years with a laser company and a space-craft firm, he joined the Laboratoire d'Optique P. M. Duffieux in 1991 as a CNRS researcher, where he initiated a research project on parametric amplification in optical fibers. He is also involved with the development of third-order nonlinear organic materials and Z-scan measurements, spatial soliton in Kerr and photorefractive media, four-wave mixing and stimulated scattering in conventional and microstructured fibers, supercontinuum generation, opto-acoustics in microstructured fibers. Since 2005, he has been working at the head of the Département d'Optique P. M. Duffieux at the FEMTO-ST Institute. 